\title{
Von Skifahren bis Tennis
}

\section{So klappt Sport mit Endoprothese}

Es werden sehr unterschiedliche Empfehlungen zur Belastbarkeit nach endoprothetischem Gelenkersatz an die Patienten gegeben. Viele von ihnen könnten Sport treiben, oft jedoch raten Ärzte ab. Inzwischen gibt es klare Empfehlungen. Allerdings handelt es sich dabei nicht um ein stur zu befolgendes Regelwerk, sagt Prof. Dr. Klaus-Peter Günther von der Klinik für Orthopädie an der Universität Dresden.

CME: Herr Prof. Günther, wie viele Patienten sind nach endoprothetischem Gelenkersatz eigentlich sportlich aktiv? Prof. Dr. Klaus-Peter Günther: In der Ulmer Arthrosestudie mit knapp 800 endoprothetisch versorgten Patienten waren fünf Jahre nach Gelenkersatz etwa $30 \%$ der Teilnehmer vergleichsweise stark sportlich aktiv. Unter den Patienten mit Hüftendoprothesen stieg der Anteil der sportlich Aktiven postoperativ im Vergleich zu präoperativ auf über $50 \%$. Bei Patienten mit Knieendoprothesen dagegen sank er von präoperativ $42 \%$ auf etwa ein Drittel.

\section{CME: Könnte es sein, dass die}

\section{Knieendoprothesenträger zu früh} ihren Gelenkersatz erhalten haben?

Günther: Nein. Gerade die Schwierigkeiten beim Sport sind ja oft mit ein Grund für den Gelenkersatz. Es gibt Vorstellungen, wonach Knieprothesenpatienten besonders vorsichtig sein müssten, was nicht unbedingt der Fall ist. Grundsätzlich besteht natürlich immer die Gefahr, dass zu früh oder zu spät operiert wird. Die Vergütungsstruktur in deutschen Kliniken fördert leider mancherorts das zu frühe Operieren. Andererseits gibt es immer noch viele Patienten, bei denen viel zu lange gewartet wird.

CME: Welche Ratschläge darf man denn heute Patienten mit Hüft- und Knieprothesen geben, was sportliche Aktivitäten anbetrifft?

Günther: Dazu sind vier Punkte wichtig. Erstens muss jeder Patient individuell beraten werden. Es ist nicht damit getan, dem Patienten eine Liste vorzulegen, welche Sportarten gut und welche schlecht sind. Individuelle Beratung heißt zu fragen: Was ist das Ziel des Patienten nach der Operation? Welche Erfahrungen hat er in der Sportart vor der Operation gemacht und wie geübt ist er? Wie fit ist der Patient ansonsten körperlich, besteht $z$. B. eine ab und zu auftretende Kreislaufschwäche? Das würde die Sturzgefahr erhöhen. Was kann der Patient sonst körperlich für sich tun? Wer in Hamburg oder Dortmund wohnt, muss ja nicht unbedingt Ski fahren. Wer jedoch in Garmisch-Partenkirchen wohnt, für den gehört das einfach dazu. Egal ob Ski alpin oder Langlauf, man sollte also erfahren sein und nicht mit diesem Sport im 70. Lebensjahr

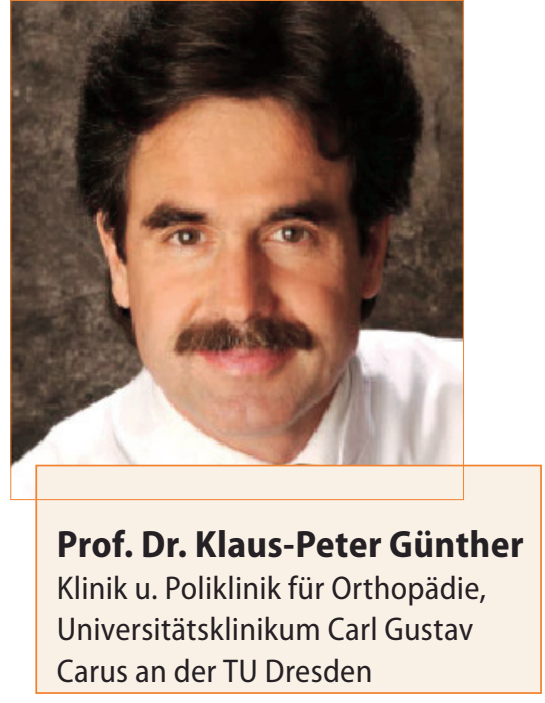

beginnen wollen. Punkt zwei: Es sollte jegliche Sportart mit erhöhter Sturzgefahr vermieden werden, die man zuvor noch nicht ausgeübt hat. Spaß, Freude und soziale Einbindung sind ebenfalls wichtige Kriterien bei der Entscheidung, denn hier geht es um Lebensqualität. Der dritte Punkt sind Stoßbelastungen. Jeder Sport, der Stoßbelastungen verursacht, ist schlechter als eine Sportart mit geringen Stoßbelastungen wie Radfahren, Bowling oder Schwimmen. Entscheidend ist aber auch, wie der Patient den Sport betreibt: Das gemütliche Doppel auf dem Tennisplatz ist absolut gesund, das kämpferische Einzel ungesund. Und schließlich viertens: Nicht übertriebene Vorsicht sollte walten, sondern der gesunde Menschenverstand. $\rightarrow$ S.44

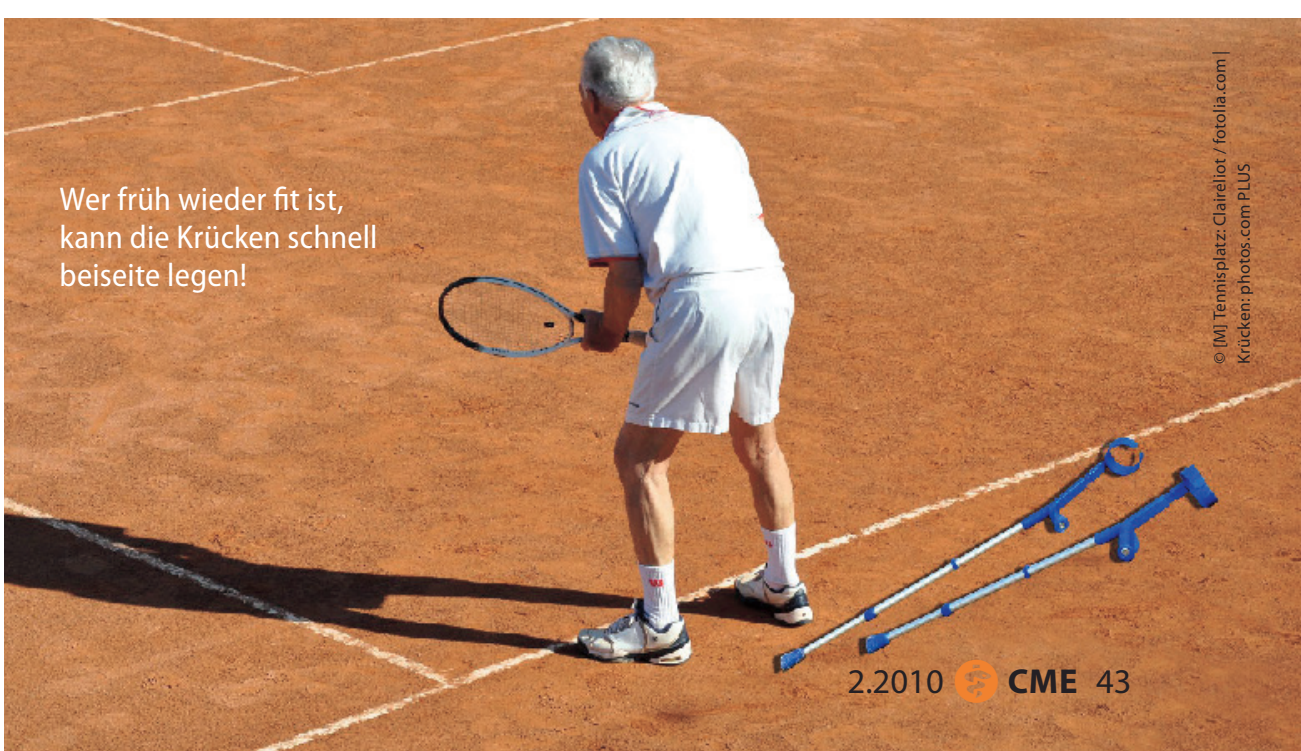

